Author affiliations and support information (if applicable) appear at the end of this article.

Published at jco.org on March 29, 2017 Clinical trial information: NCT02000427.

Corresponding author: Giovanni Martinelli, MD, Institute of Hematology and Medical Oncology "L. and A. Seràgnoli", S. Orsola University Hospital, Via Massarenti, 9-40138, Bologna, Italy; e-mail: giovanni. martinelli2@unibo.it.

(c) 2017 by American Society of Clinical Oncology

$0732-183 X / 17 / 3516 w-1795 w / \$ 20.00$

\section{Complete Hematologic and Molecular Response in Adult Patients With Relapsed/Refractory Philadelphia Chromosome-Positive B-Precursor Acute Lymphoblastic Leukemia Following Treatment With Blinatumomab: Results From a Phase II, Single-Arm, Multicenter Study}

Giovanni Martinelli, Nicolas Boissel, Patrice Chevallier, Oliver Ottmann, Nicola Gökbuget, Max S. Topp, Adele K. Fielding, Alessandro Rambaldi, Ellen K. Ritchie, Cristina Papayannidis, Lulu Ren Sterling, Jonathan Benjamin, and Anthony Stein

\title{
Purpose
}

Few therapeutic options are available for patients with Philadelphia chromosome-positive $\left(\mathrm{Ph}^{+}\right)$ B-precursor acute lymphoblastic leukemia (ALL) who progress after failure of tyrosine kinase inhibitor (TKI) - based therapy. Here, we evaluated the efficacy and tolerability of blinatumomab in patients with relapsed or refractory $\mathrm{Ph}^{+} \mathrm{ALL}$.

\section{Patients and Methods}

This open-label phase II study enrolled adults with $\mathrm{Ph}^{+} \mathrm{ALL}$ who had relapsed after or were refractory to at least one second-generation or later TKI or were intolerant to second-generation or later TKIs and intolerant or refractory to imatinib. Blinatumomab was administered in 28-day cycles by continuous intravenous infusion. The primary end point was complete remission (CR) or CR with partial hematologic recovery (CRh) during the first two cycles. Major secondary end points included minimal residual disease response, rate of allogeneic hematopoietic stem-cell transplantation, relapse-free survival, overall survival, and adverse events (AEs).

\section{Results}

Of 45 patients, 16 (36\%; 95\% Cl, 22\% to 51\%) achieved CR/CRh during the first two cycles, including four of 10 patients with the T315I mutation; 88\% of CR/CRh responders achieved a complete minimal residual disease response. Seven responders (44\%) proceeded to allogeneic hematopoietic stem-cell transplantation, including 55\% (six of 11) of transplantation-naïve responders. Median relapse-free survival and overall survival were 6.7 and 7.1 months, respectively. The most frequent AEs were pyrexia (58\%), febrile neutropenia (40\%), and headache (31\%). Three patients had cytokine release syndrome (all grade 1 or 2), and three patients had grade 3 neurologic events, one of which (aphasia) required temporary treatment interruption. There were no grade 4 or 5 neurologic events.

\section{Conclusion}

Single-agent blinatumomab showed antileukemia activity in high-risk patients with $\mathrm{Ph}^{+} \mathrm{ALL}$ who had relapsed or were refractory to TKIs. AEs were consistent with previous experience in $\mathrm{Ph}^{-} \mathrm{ALL}$.

\section{J Clin Oncol 35:1795-1802. (C) 2017 by American Society of Clinical Oncology}

\section{INTRODUCTION}

Philadelphia chromosome-positive $\left(\mathrm{Ph}^{+}\right)$acute lymphoblastic leukemia (ALL) constitutes approximately $25 \%$ of adult B-precursor ALL and is characterized by a reciprocal $t(9 ; 22)$ translocation that generates a chimeric fusion protein, BCRABL1, with dysregulated tyrosine kinase activity.
The presence of the Philadelphia chromosome has historically been associated with a very poor prognosis, but response and survival outcomes have significantly improved over the last decade. The addition of targeted tyrosine kinase inhibitors (TKIs) to induction and consolidation chemotherapy followed by allogeneic hematopoietic stem-cell transplantation (alloHSCT) has yielded $>90 \%$ complete remission $(\mathrm{CR})^{1}$ and up to 
$60 \% 5$-year overall survival (OS) rates. $^{2-4}$ TKIs have also permitted de-escalation of chemotherapy without loss of response or event-free survival in older patients, and deep molecular responses may be achieved in some patients. ${ }^{4}$ A retrospective analysis in the posttransplantation setting has shown that use of prophylactic TKIs is associated with a significantly lower incidence of relapse among patients with $\mathrm{Ph}^{+}$ALL. ${ }^{5}$ However, relapse remains clinically challenging and is frequently associated with resistance substitutions in the ABL kinase domain, particularly within the threonine 315 residue (T315I). ${ }^{6,7}$ Although alternative TKIs for salvage therapy may be effective in some patients, ${ }^{8,9}$ remission is short. ${ }^{10,11}$ In the first-line setting and in the context of alloHSCT, minimal residual disease (MRD), as assessed by reverse transcription quantitative polymerase chain reaction (RT-qPCR), may provide additional prognostic information for relapse. ${ }^{12-14}$ There is no current evidence of long-term survival mediated by TKIs after relapse. ${ }^{11}$ Sequential TKI exposure can permit emergence of compound mutations that eventually confer resistance to all TKIs. ${ }^{15-17}$ Relapse can also occur without detection of $B C R-A B L 1$ mutations, suggesting that there are kinase-independent pathways to leukemic cell survival and proliferation. Therefore, alternative approaches beyond kinase inhibition that increase the potential for achieving CR and longer OS are needed in relapsed/refractory (R/R) $\mathrm{Ph}^{+}$ALL.

Blinatumomab is a bispecific T-cell engager antibody construct that is designed to direct cytotoxic $\mathrm{T}$ cells to CD19expressing $\mathrm{B}$ cells. ${ }^{18}$ A large phase II study confirmed antileukemia activity and tolerability of blinatumomab in adults with $\mathrm{R} / \mathrm{R} \mathrm{Ph}^{-} \mathrm{ALL}$ and reported $43 \% \mathrm{CR}$ or CR with partial hematologic recovery (CRh), 82\% MRD response, and relapse-free survival (RFS) of 5.9 months. ${ }^{19}$ Patients with $\mathrm{Ph}^{+}$ALL are distinct from those with $\mathrm{Ph}^{-}$ALL and often have different baseline demographics that may have a significant impact on clinical outcomes. Here, we evaluated the efficacy and tolerability of single-agent blinatumomab in patients with $\mathrm{R} / \mathrm{R} \mathrm{Ph}^{+}$ALL who progressed after or were intolerant to a second-generation or later TKI.

\section{PATIENTS AND METHODS}

\section{Patients}

This was an open-label, single-arm, multicenter, phase II study of blinatumomab in adults with $\mathrm{R} / \mathrm{R} \mathrm{Ph}^{+} \mathrm{ALL}$ at 19 European and US centers. Eligible adults (age $\geq 18$ years) had $\mathrm{Ph}^{+}$B-precursor ALL, had relapsed after or were refractory to at least one second-generation or later TKI (dasatinib, nilotinib, bosutinib, ponatinib), or were intolerant to secondgeneration or later TKIs and intolerant or refractory to imatinib. The Philadelphia chromosome was detected by cytogenetics, fluorescence in situ hybridization, and/or BCR-ABL1 PCR at individual study sites. Additional eligibility requirements included $>5 \%$ bone marrow blasts as determined by a central laboratory and an Eastern Cooperative Oncology Group status $\leq 2$. Key exclusion criteria were alloHSCT within 12 weeks before the start of blinatumomab treatment, active acute or chronic (grade 2 to 4) graft-versus-host disease, systemic treatment of graft-versus-host disease within 2 weeks before treatment start, history or presence of clinically relevant CNS pathology, active CNS ALL, and isolated extramedullary disease. Any TKI therapy, antitumor therapy other than blinatumomab, chronic systemic high-dose corticosteroid therapy, or other immunosuppressive therapies were prohibited during treatment. Each center's institutional review board or ethics committee approved the study; all patients provided written informed consent.

\section{Study Procedures}

Patients received blinatumomab as a continuous intravenous infusion at fixed stepwise doses $(9 \mu \mathrm{g} /$ day in week 1 of cycle 1 followed by $28 \mu \mathrm{g} /$ day thereafter). ${ }^{19,20}$ Each treatment cycle included 4 weeks of continuous intravenous infusion followed by a 2 -week treatment-free interval. Patients received two initial cycles of blinatumomab to induce remission. CR was defined as $\leq 5 \%$ bone marrow blasts, platelets $>100,000 / \mu \mathrm{L}$, and absolute neutrophil count $>1,000 / \mu \mathrm{L}$; CRh was defined as $\leq 5 \%$ bone marrow blasts, platelets $>50,000 / \mu \mathrm{L}$, and absolute neutrophil count $>500 / \mu \mathrm{L}$. If CR/CRh was achieved, the patient could receive up to three additional cycles of blinatumomab as consolidation therapy, unless alloHSCT was scheduled earlier.

To reduce tumor burden and cytokine release syndrome (CRS), patients with a high baseline blast count as determined by a local laboratory ( $>50 \%$ bone marrow blasts or $\geq 15,000 / \mu \mathrm{L}$ peripheral blast count) received prephase treatment with dexamethasone $10 \mathrm{mg} / \mathrm{m}^{2}$ per day (for up to 5 days) up to a maximum of $24 \mathrm{mg} /$ day (absolute). All patients were given dexamethasone $20 \mathrm{mg}$ intravenously 1 hour before each cycle and dose step and within 1 hour before restarting treatment for dose interruptions. For dose interruptions that resulted from a neurologic event, patients received dexamethasone at least $24 \mathrm{mg} /$ day, with stepwise reductions over 4 days. Intrathecal chemoprophylaxis (eg, methotrexate 12 to $15 \mathrm{mg}$, cytarabine $40 \mathrm{mg}$, and dexamethasone $4 \mathrm{mg}$ or equivalent) was required before initiation of treatment and at the end of treatment cycles. Detailed descriptions regarding blinatumomab dose modifications, interruptions, and discontinuation are provided in the Appendix (online only).

Hematologic and molecular responses were assessed by bone marrow aspirate or biopsy on day 29 of each cycle by using a central reference laboratory. MRD response was determined by $B C R-A B L 1$ quantification only for patients achieving CR/CRh. Complete MRD response was defined as no detectable $B C R-A B L 1$ transcripts by allele-specific real-time qPCR with an internal $A B L$ amplification control, as established by a central laboratory (LabCorp, Burlington, NC; assay sensitivity $\geq 10^{-5} ; 0.00 \%$ $B C R-A B L 1 / A B L 1)$. $p 190$ and $p 210$ designation was based on determination of e1a2 (p190) or b2a2/b3a2 (p210) transcript levels from RT-qPCR performed on pretreatment bone marrow aspirates. ${ }^{21}$ Adverse events (AEs) and serious AEs, which were recorded from treatment start until at least 30 days after treatment end, were graded according to the Common Terminology Criteria for Adverse Events (CTCAE) v4.0. ${ }^{22}$

\section{Statistical Methods}

The primary end point was the proportion of patients who achieved $\mathrm{CR} / \mathrm{CRh}$ during the first two cycles of blinatumomab treatment. Sample size was calculated for a Simon's two-stage design ${ }^{23}$ based on a one-sided type I error of 0.025 and a power of $90 \%$ to detect the effective response rate assumption of $\geq 30 \%$ over an ineffective treatment rate of $\leq 10 \%$.

Major secondary end points included MRD response rate during the first two cycles of treatment, RFS, OS, and alloHSCT after blinatumomabinduced remission. RFS was measured from the time of first CR/CRh to hematologic or extramedullary relapse or death resulting from any cause. OS was measured from the time of first blinatumomab dose to death resulting from any cause. Kaplan-Meier methods were used to estimate probability of RFS and OS over time; OS among MRD responders was evaluated using the landmark analysis approach to minimize bias that patients must survive to their first MRD assessment to be included.

\section{RESULTS}

\section{Patient Characteristics}

Between January 3, 2014, and May 20, 2015, 45 patients were enrolled and treated with blinatumomab. Median age was 55 years (range, 23 to 78 years). Forty-four percent of patients had relapsed after prior alloHSCT. Median baseline bone marrow blast percentage was $80 \%$ (range, $6 \%$ to $98 \%$ ). At data cutoff (May 20, 
2015), 23 patients (51\%) remained in the study (21 were in followup, and two were still receiving treatment).

Eighty-four percent of patients had received $\geq 2$ prior TKIs; $51 \%$ had received prior ponatinib (third-generation TKI; Table 1). All patients were either refractory to $(56 \%)$, had relapsed on (33\%), or exhibited disease progression after (11\%) their prior TKI therapy; no patient had TKI intolerance listed as the reason for discontinuation of their prior TKI therapy. One patient had ALL resistant to imatinib and was never exposed to a second-generation or later TKI (protocol deviation).

Fifty-nine percent of patients had additional cytogenetic abnormalities. A subset of patients $(\mathrm{n}=10)$ had both $p 190$ and p210 transcripts, although in all patients, p210 was 2.1- to 3.5-log higher and thus designated as $p 210$. Of 37 patients evaluable for TKI mutational analysis, $46 \%$ had $A B L 1$ kinase domain mutations, including the T315I mutation (27\%; Table 1).

\section{Response}

The median number of blinatumomab cycles received was two (range, one to five). Thirty-six percent (95\% CI, 22\% to $51 \%$ ) of

Table 1. Patient Demographic and Clinical Characteristics at Baseline

\begin{tabular}{|c|c|c|}
\hline \multirow[b]{2}{*}{ Characteristic } & \multicolumn{2}{|c|}{ Patients ( $N=45$ ) } \\
\hline & No. & $\%$ \\
\hline \multicolumn{3}{|l|}{ Sex } \\
\hline Male & 24 & 53 \\
\hline Female & 21 & 47 \\
\hline Median age, years (range) & \multicolumn{2}{|c|}{$55(23-78)$} \\
\hline \multicolumn{3}{|l|}{ Age group, years } \\
\hline 18 to $<55$ & 22 & 49 \\
\hline$\geq 55$ & 23 & 51 \\
\hline \multicolumn{3}{|l|}{ Cytogenetics and molecular analyses* } \\
\hline $\begin{array}{l}\text { Philadelphia chromosome and other } \\
\text { cytogenetic abnormalities }\end{array}$ & $22 / 38$ & 58 \\
\hline ABL1 kinase domain mutations & $17 / 37$ & 46 \\
\hline T315I mutation & $10 / 37$ & 27 \\
\hline \multicolumn{3}{|l|}{ No. of prior TKI treatmentst } \\
\hline 1 & 7 & 16 \\
\hline 2 & 21 & 47 \\
\hline 3 & 13 & 29 \\
\hline 4 & 4 & 9 \\
\hline Prior TKI‡ & 45 & 100 \\
\hline Imatinib & 25 & 56 \\
\hline Dasatinib & 39 & 87 \\
\hline Nilotinib & 16 & 36 \\
\hline Ponatinib & 23 & 51 \\
\hline \multicolumn{3}{|l|}{ Prior alloHSCT } \\
\hline Yes & 20 & 44 \\
\hline No & 25 & 56 \\
\hline \multicolumn{3}{|l|}{ Bone marrow blasts (central review) } \\
\hline$<10 \%$ & 2 & 4 \\
\hline $10 \%$ to $<50 \%$ & 9 & 20 \\
\hline $50 \%$ to $<75 \%$ & 6 & 13 \\
\hline$\geq 75 \%$ & 28 & 62 \\
\hline
\end{tabular}

Abbreviations: ABL1, Abelson murine leukemia viral oncogene homolog 1; alloHSCT, allogeneic hematopoietic stem-cell transplantation; TKI, tyrosine kinase inhibitor.

*Philadelphia chromosome was detected by cytogenetics/metaphase spread, fluorescence in situ hybridization, or $B C R-A B L 1$ polymerase chain reaction. Numerators indicate the number of patients in each subgroup, and denominators indicate the number of evaluable patients tested by a specific methodology. tOne patient had acute lymphoblastic leukemia that was resistant to imatinib and was never exposed to a second-generation or later TKI (protocol deviation). $\ddagger$ Prior TKI use was not mutually exclusive. patients $(\mathrm{n}=16)$ achieved CR/CRh within the first two cycles, with most patients $(\mathrm{n}=14)$ achieving CR (Table 2). Among evaluable responders in cycle 1, 10 achieved CR before initiation of a second cycle, two achieved CRh, and two were without hematologic recovery. Two patients had inevaluable responses at the end of cycle 1; however, both achieved blast clearance by the end of cycle 2 (one with $\mathrm{CR}$ and one with $\mathrm{CRh}$ ). Among nonresponders or those with progressive disease at the end of cycle 1, two achieved CR with incomplete hematologic recovery $(\mathrm{CRi})$ at the end of cycle 2 . The one patient who never received a second-generation or later TKI (protocol deviation) did not respond to treatment. Twelve (86\%) of the 14 $\mathrm{CR}$ responders and both patients with $\mathrm{CRh}$ also achieved a complete MRD response; the remaining two responders had persistent measurable MRD and relapsed during subsequent cycles of therapy.

Patients achieved responses to blinatumomab regardless of prior TKI therapy: $47 \%$ had three or more prior TKIs and $35 \%$ had received prior ponatinib. Similarly, responses were also observed regardless of ABL1 kinase domain mutational status (Fig 1). Specifically, among the 10 patients with the T315I mutation (nine of whom had received prior ponatinib), four (40\%) responded, with all four CR/CRh responders also achieving a complete MRD response.

Subgroup analyses of response showed no statistically significant differences based on collected baseline features, including age and prior therapies, but the largest difference in median $\mathrm{CR} / \mathrm{CRh}$ response was observed between the subgroups of $<50 \%$ and $\geq 50 \%$ bone marrow blasts at baseline (64\% [95\% CI, $31 \%$ to $89 \%$ ] and $27 \%$ [ $95 \%$ CI, $13 \%$ to $44 \%$ ], respectively; Fig 1).

\section{Survival}

Median RFS was 6.7 months (95\% CI, 4.4 to NE [not estimable] months), with a median follow-up of 9.0 months. Median RFS among the 14 patients who achieved a complete MRD response was 6.8 months (95\% CI, 4.4 to NE months) with a median follow-up of 9.0 months, which was similar to that of the entire CR/CRh responder patient population; RFS was not significantly impacted when censoring for alloHSCT (Fig 2A). No difference in median RFS was observed between responders younger than age 55 years (5.5 months; 95\% CI, 3.6 to NE months) and adults age 55 years or older (6.7 months; $95 \%$ CI, 3.8 to NE months). Of the 16 responders, seven $(44 \%)$ were alive without relapse, eight $(50 \%)$ had relapsed with a median time to relapse of 6.7 months (95\% CI, 4.4 to NE months), and one patient died in CR after alloHSCT (133 days after achieving CR). Of the patients who had relapsed, three had relapsed during treatment (including two CR responders who did not achieve a complete MRD response), two relapsed without receiving alloHSCT, and three relapsed after receiving alloHSCT.

Median OS was 7.1 months (95\% CI, 5.6 to NE months) with or without censoring for alloHSCT (Fig 2B), with a median follow-up of 8.8 months. Using a landmark OS analysis (landmark time point at the end of cycle 2 of blinatumomab treatment), median OS was not reached for the 18 patients who achieved a complete MRD response, with a median follow-up of 5.3 months. Among MRD nonresponders, the median OS was 3.9 months (95\% CI, 3.0 to NE months).

\section{alloHSCT}

Of the $16 \mathrm{CR} / \mathrm{CRh}$ responders, seven (44\%) proceeded to alloHSCT, including four who remained in continuous 


\begin{tabular}{|c|c|c|c|}
\hline Outcome & Patients (n/N1)* & Proportion (\%) & $95 \% \mathrm{Cl}$ \\
\hline \multicolumn{4}{|l|}{ Primary end point } \\
\hline CR/CRh during the first two cycles & $16 / 45$ & 36 & 22 to 51 \\
\hline $\begin{array}{l}\text { Philadelphia chromosome and other cytogenetic } \\
\text { abnormalities }\end{array}$ & $10 / 22$ & 45 & 24 to 68 \\
\hline ABL1 kinase domain mutations & $6 / 17$ & 35 & 14 to 62 \\
\hline T315I mutation & $4 / 10$ & 40 & 12 to 74 \\
\hline p190 BCR/ABL1 isoform $t$ & $10 / 26$ & 39 & 20 to 59 \\
\hline p210 BCR/ABL1 isoform $t$ & $5 / 16$ & 31 & 11 to 59 \\
\hline \multicolumn{4}{|l|}{ No. of prior TKI therapies } \\
\hline 1 & $1 / 7$ & 14 & $<1$ to 58 \\
\hline 2 & $7 / 21$ & 33 & 15 to 57 \\
\hline$\geq 3$ & $8 / 17$ & 47 & 23 to 72 \\
\hline Prior ponatinib & $8 / 23$ & 35 & 16 to 57 \\
\hline Prior alloHSCT & $5 / 20$ & 25 & 9 to 49 \\
\hline Age 18 to $<55$ years & $8 / 22$ & 36 & 17 to 59 \\
\hline Age $\geq 55$ years & $8 / 23$ & 35 & 16 to 57 \\
\hline Bone marrow blasts $<50 \%$ & $7 / 11$ & 64 & 31 to 89 \\
\hline Bone marrow blasts $\geq 50 \%$ & $9 / 34$ & 27 & 13 to 44 \\
\hline \multicolumn{4}{|l|}{ Secondary end points } \\
\hline \multicolumn{4}{|l|}{ Best response during the first two cycles } \\
\hline $\mathrm{CR}$ & $14 / 45$ & 31 & 18 to 47 \\
\hline $\mathrm{CRh}$ & $2 / 45$ & 4 & 1 to 15 \\
\hline Complete MRD responseł & $14 / 16$ & 88 & 62 to 98 \\
\hline alloHSCT after blinatumomab-induced remission§ & $4 / 16$ & 25 & 7 to 52 \\
\hline Age 18 to $<55$ years & $2 / 8$ & 25 & 3 to 65 \\
\hline Age $\geq 55$ years & $2 / 8$ & 25 & 3 to 65 \\
\hline 100-day post-transplant mortality rate§ & $1 / 4$ & 25 & 4 to 87 \\
\hline \multicolumn{4}{|c|}{ 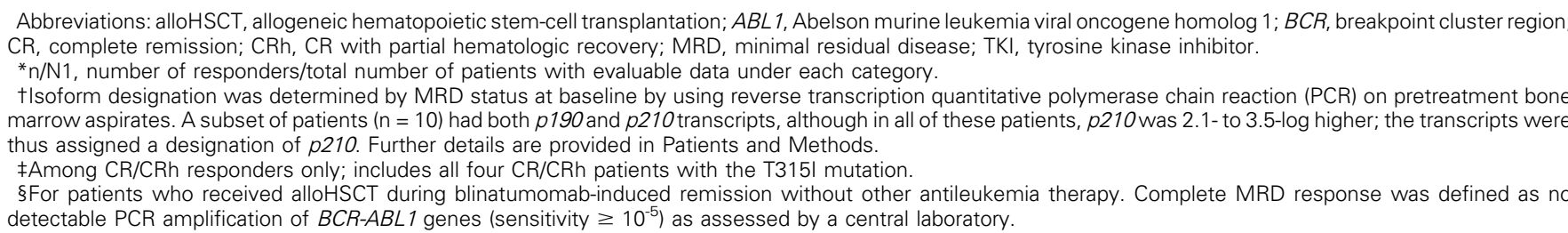 } \\
\hline
\end{tabular}

blinatumomab-induced remission without other antileukemia therapy and three who received blinatumomab with other antileukemia therapy. Of the latter three patients, one received other anticancer therapy prior to alloHSCT and remained in remission, and two had relapsed before proceeding to alloHSCT. The 100-day mortality was $25 \%$ (one death) among the four patients who received alloHSCT while in blinatumomab-induced remission (Table 2). One responder received a second alloHSCT after blinatumomab-induced remission.

\section{AEs}

Among the 45 patients who received blinatumomab, the most common treatment-emergent AEs included pyrexia (58\%), febrile neutropenia $(40 \%)$, and headache $(31 \%)$ (Table 3$)$. Thirty-seven patients $(82 \%)$ had grade $\geq 3$ treatment-emergent AEs, the most frequent of which were febrile neutropenia $(27 \%)$, thrombocytopenia $(22 \%)$, and anemia $(16 \%)$. Twenty patients $(44 \%)$ had grade $\geq 3$ treatment-emergent AEs that were considered possibly related to blinatumomab (per investigator's assessment), most commonly febrile neutropenia and increased levels of alanine aminotransferase (11\% each). Thirty-three patients $(73 \%)$ had grade $3 \mathrm{AEs}$, and 16 patients (36\%) had grade 4 AEs. Five patients (none of whom achieved CR/CRh) had fatal AEs: multiorgan failure (age 55 years), infection (sepsis, age 40 years; septic shock, age 33 years), cerebral hemorrhage (age 25 years), and respiratory failure (age 42 years). One fatal AE (septic shock) was considered treatment-related by the investigator. This patient had disease persistence and died 13 days after protocol-directed discontinuation of blinatumomab.

CRS occurred in three patients (all grade 1 or 2), but none of the CRS events resulted in treatment discontinuation or interruption. Twenty-one patients (47\%) experienced neurologic events, most commonly paresthesia $(13 \%)$, confusional state (11\%), dizziness $(9 \%)$, and tremor (9\%; Table 3$)$. Three patients had grade 3 neurologic events (aphasia, hemiplegia, and nervous system disorder or depressed level of consciousness). However, only one patient required treatment interruption (aphasia). No patients had grade 4 or 5 neurologic events. All but one of the grade 3 neurologic events resolved, with a maximum duration of 15 days.

\section{DISCUSSION}

In this primary analysis of a phase II study in adults with heavily pretreated $\mathrm{R} / \mathrm{R} \mathrm{Ph}^{+} \mathrm{ALL}$, treatment with single-agent blinatumomab resulted in a $36 \% \mathrm{CR} / \mathrm{CRh}$ rate within the first two cycles, 


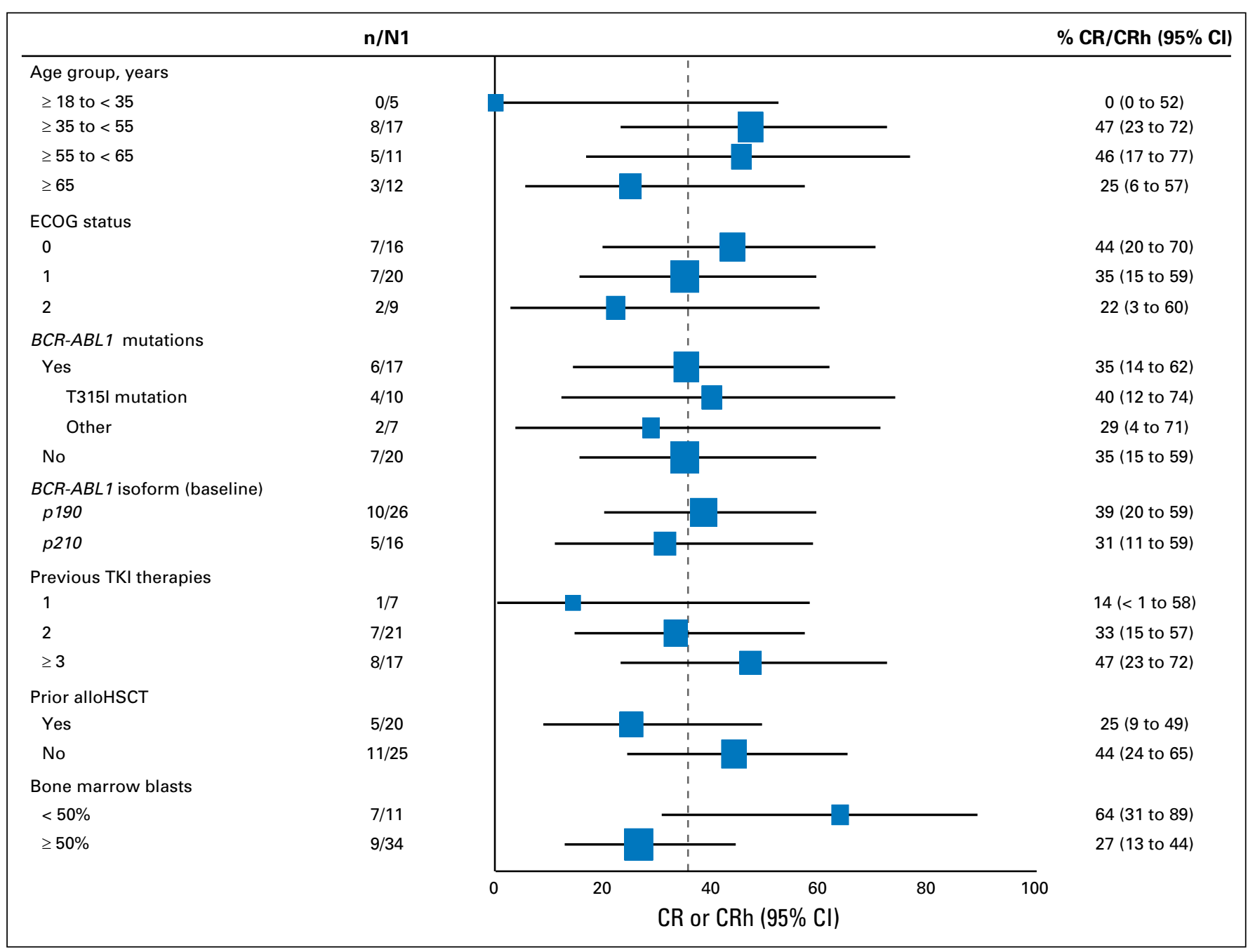

Fig 1. Overall responses (complete remission/complete remission with partial hematologic recovery [CR/CRh]) during the first two treatment cycles among prespecified patient subgroups. The dashed line represents the point estimate for $\mathrm{CR} / \mathrm{CRh}$ for the entire patient population, and the box size indicates the relative population weight. n/N1, number of responders/total number of patients with evaluable responses within each category. alloHSCT, allogeneic hematopoietic stem-cell transplantation; ECOG, Eastern Cooperative Oncology Group; TKI, tyrosine kinase inhibitor.

with $88 \%$ of responders also achieving a complete MRD response. Median RFS and OS were 6.7 and 7.1 months, respectively. AEs were consistent with those previously observed for blinatumomab in the setting of $\mathrm{R} / \mathrm{R} \mathrm{Ph}^{-}$ALL. As predicted by the mechanism of action of blinatumomab, these data demonstrate that hematologic and molecular responses were independent of $B C R-A B L 1$ status, including the presence of the T315I mutation. More than one third of patients $(38 \%)$ had at least one $B C R-A B L 1$ mutation, including 10 with the T315I mutation, a recognized negative prognostic factor, even for treatment with ponatinib and/or alloHSCT. ${ }^{24,25}$ In fact, the T315I mutation along with additional kinase domain mutations have been associated with poor treatment responses and outcomes, possibly as a result of increased genetic heterogeneity among leukemic subclones that may lead to TKI-based drug resistance. $^{26}$

All patients had heavily pretreated $\mathrm{R} / \mathrm{R} \mathrm{Ph}^{+} \mathrm{ALL}$, with nearly half $(47 \%)$ having failed two prior TKI treatments and one third $(38 \%)$ having failed three or more prior TKI treatments; $62 \%$ had $\geq 75 \%$ blasts at baseline; or $44 \%$ had relapsed after alloHSCT. Notably, these data show that the proportion of patients who achieved CR/CRh was in line with that reported for less heavily pretreated populations or those in the $\mathrm{Ph}^{-}$setting (36\% v 43\% in $\mathrm{Ph}^{-} \mathrm{ALL}$ ), with similar or better response duration than that observed with TKI therapies. ${ }^{27-29}$ Although not statistically significant, a higher CR/CRh rate was observed in patients with a low $(<50 \%)$ blast percentage at baseline compared with patients who had a high $(\geq 50 \%)$ blast percentage (Fig 1), a trend consistent with blinatumomab treatment in the $\mathrm{Ph}^{-}$setting. ${ }^{19}$ Interestingly, the five patients younger than age 35 years did not respond, which may be the result of higher disease burden (eg, $\geq 50 \%$ bone marrow blasts) and disease aggressiveness (eg, shorter median time from initial diagnosis to treatment and from first prior alloHSCT to relapse) among these patients. Given the very small number of patients, however, this is highly exploratory and speculative. Tumor load may be a marker of disease aggressiveness of leukemia, which may or may not be modified by cytoreduction. Alternatively, any potential association between tumor load and response may 


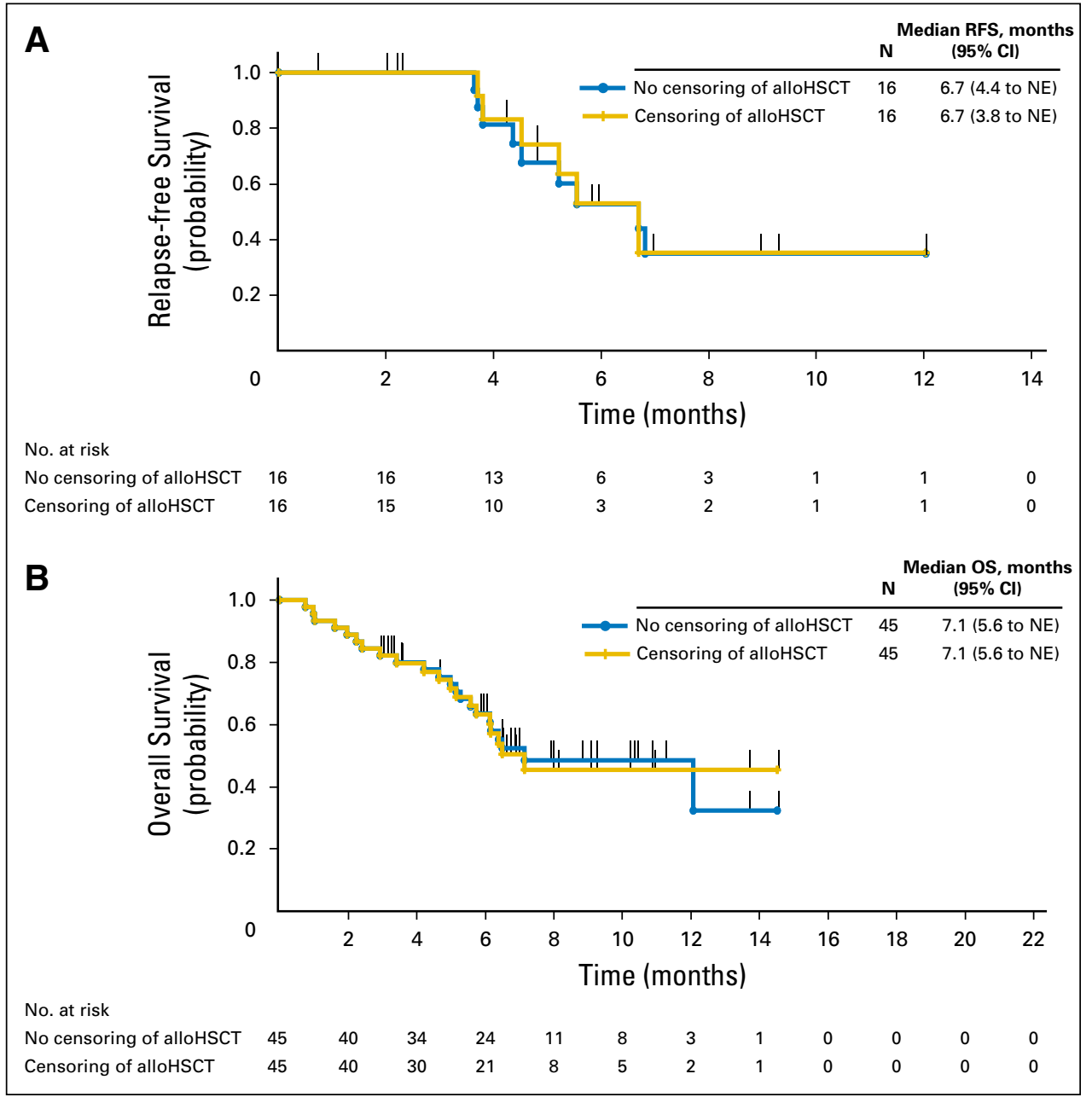

Fig 2. (A) Relapse-free survival (RFS) and (B) overall survival (OS) with and without censoring at time of allogeneic hematopoietic stem-cell transplantation (alloHSCT). NE, not estimable.

reflect an unfavorable effector-to-target ratio, which could be amenable to intervention. In a randomized trial of blinatumomab versus standard chemotherapy for $\mathrm{R} / \mathrm{R} \mathrm{Ph}^{-} \mathrm{ALL}$, the survival benefit of blinatumomab was observed even among patients with high bone marrow blasts. ${ }^{30}$

Therapeutic options for patients with $\mathrm{R} / \mathrm{R} \mathrm{Ph}^{+}$ALL are scarce and restricted to untargeted therapies such as combination chemotherapy, interferon-alpha, or omacetaxine mepesuccinate, which provide only short-term and primarily hematologic responses with poor cytogenetic efficacy. ${ }^{31-33}$ For eligible patients with $\mathrm{Ph}^{+}$ALL and the T315I mutation, alloHSCT remains the best treatment option, providing acceptable OS rates and, in some cases, long-term control of the malignancy. Because of its TKIindependent mechanism of action, blinatumomab can overcome the T315I resistance mechanism to induce directed immunemediated cell death of $\mathrm{Ph}^{+}$blasts. However, it is unknown whether $B C R-A B L 1$-independent signaling in patients with ALL that is refractory to TKIs may also predispose patients to be resistant to blinatumomab treatment. Here, response rates to blinatumomab and RFS were found to be similar between younger (age younger than 55 years) and older (age 55 years or older) adults, which was also reported for $\mathrm{Ph}^{-} \mathrm{ALL},{ }^{34}$ suggesting a benefit for older adults who are typically ineligible for alloHSCT or have increased comorbidities. Moreover, blinatumomab induced complete MRD responses in $88 \%$ of $\mathrm{Ph}^{+}$patients who achieved $\mathrm{CR} / \mathrm{CRh}$, suggesting that blinatumomab is an effective bridge to transplantation.

Because duration of response to TKIs is short in $\mathrm{Ph}^{+} \mathrm{ALL}^{35}$ consolidative alloHSCT remains the standard of care for eligible patients, and achievement of CR and MRD response is desirable for stem-cell transplantation. $\mathrm{Ph}^{+}$patients, such as those treated in this trial, tend to be older and likely ineligible for myeloablative conditioning regimens. ${ }^{36}$ However, these patients may be eligible to receive reduced-intensity conditioning regimens, and recent reports suggest that MRD status has prognostic relevance in this setting, presumably because of greater reliance on graft-versusleukemia effects and less reliance on cytoreduction after the conditioning regimen. ${ }^{8,13,37}$ Recurrence of leukemia after transplantation in patients with incomplete MRD response also raises the necessity for rigorous and standard MRD monitoring of these patients, as is currently done in pediatric and cooperative studies in the adult ALL setting. ${ }^{38,39}$

In summary, these data demonstrate efficacy of single-agent blinatumomab in $\mathrm{R} / \mathrm{R} \mathrm{Ph}^{+} \mathrm{ALL}$, suggesting a possible future role in Ph-like ALL and combination therapies with TKIs for this disease setting. 
Table 3. AEs and Neurologic Events (regardless of causality)

\begin{tabular}{|c|c|c|c|c|c|c|c|c|}
\hline \multirow[b]{3}{*}{ Event } & \multicolumn{8}{|c|}{ Grade } \\
\hline & \multicolumn{2}{|c|}{ Any } & \multicolumn{2}{|c|}{1 to 2} & \multicolumn{2}{|c|}{3} & \multicolumn{2}{|c|}{4} \\
\hline & No. & $\%$ & No. & $\%$ & No. & $\%$ & No. & $\%$ \\
\hline Patients with AEs & 45 & 100 & 45 & 100 & 33 & 73 & 16 & 36 \\
\hline \multicolumn{9}{|c|}{ AEs of grade $\geq 3$ occurring in $\geq 5 \%$ of patients* } \\
\hline Pyrexia & 26 & 58 & 24 & 53 & 5 & 11 & 0 & 0 \\
\hline Febrile neutropenia & 18 & 40 & 9 & 20 & 12 & 27 & 0 & 0 \\
\hline Headache & 14 & 31 & 13 & 29 & 3 & 7 & 0 & 0 \\
\hline Anemia & 13 & 29 & 9 & 20 & 7 & 16 & 1 & 2 \\
\hline Thrombocytopenia & 10 & 22 & 4 & 9 & 5 & 11 & 7 & 16 \\
\hline Pain & 7 & 16 & 4 & 9 & 4 & 9 & 0 & 0 \\
\hline Increased aspartate aminotransferase & 6 & 13 & 3 & 7 & 3 & 7 & 2 & 4 \\
\hline Increased alanine aminotransferase & 5 & 11 & 1 & 2 & 5 & 11 & 0 & 0 \\
\hline Device-related infection & 5 & 11 & 3 & 7 & 3 & 7 & 0 & 0 \\
\hline Neutropenia & 3 & 7 & 0 & 0 & 0 & 0 & 3 & 7 \\
\hline Patients with neurologic events & 21 & 47 & 20 & 44 & 3 & 7 & 0 & 0 \\
\hline \multicolumn{9}{|c|}{ Neurologic events occurring in two or more patients } \\
\hline Paresthesia & 6 & 13 & 6 & 13 & 0 & 0 & 0 & 0 \\
\hline Confusional state & 5 & 11 & 5 & 11 & 0 & 0 & 0 & 0 \\
\hline Dizziness & 4 & 9 & 4 & 9 & 0 & 0 & 0 & 0 \\
\hline Tremor & 4 & 9 & 4 & 9 & 0 & 0 & 0 & 0 \\
\hline Aphasia & 2 & 4 & 1 & 2 & 1 & 2 & 0 & 0 \\
\hline Cerebellar syndrome & 2 & 4 & 2 & 4 & 0 & 0 & 0 & 0 \\
\hline Memory impairment & 2 & 4 & 2 & 4 & 0 & 0 & 0 & 0 \\
\hline Nervous system disorder & 2 & 4 & 1 & 2 & 1 & 2 & 0 & 0 \\
\hline
\end{tabular}

Abbreviation: $A E$, adverse event.

${ }^{*}$ Cutoff based on grade $\geq 3$ AEs.

\section{AUTHORS' DISCLOSURES OF POTENTIAL CONFLICTS OF INTEREST}

Disclosures provided by the authors are available with this article at jco.org.

\section{AUTHOR CONTRIBUTIONS}

Conception and design: Max S. Topp, Jonathan Benjamin
Provision of study materials or patients: Nicolas Boissel, Oliver Ottmann, Ellen K. Ritchie

Collection and assembly of data: Giovanni Martinelli, Oliver Ottmann, Nicola Gökbuget, Max S. Topp, Adele K. Fielding, Alessandro Rambaldi, Ellen K. Ritchie, Cristina Papayannidis, Jonathan Benjamin

Data analysis and interpretation: Nicolas Boissel, Patrice Chevallier, Oliver Ottmann, Max S. Topp, Adele K. Fielding, Ellen K. Ritchie, Lulu Ren Sterling, Jonathan Benjamin, Anthony Stein

Manuscript writing: All authors

Final approval of manuscript: All authors Accountable for all aspects of the work: All authors

\section{REFERENGES}

1. Fielding AK: Current treatment of Philadelphia chromosome-positive acute lymphoblastic leukemia. Hematology (Am Soc Hematol Educ Program) 2011: 231-237, 2011

2. Fielding AK: Treatment of Philadelphia chromosome-positive acute lymphoblastic leukemia in adults: A broader range of options, improved outcomes, and more therapeutic dilemmas. Am Soc Clin Oncol Educ Book 2015:e352-e359, 2015

3. Tanguy-Schmidt $A$, Rousselot $P$, Chalandon $Y$, et al: Long-term follow-up of the imatinib GRAAPH2003 study in newly diagnosed patients with de novo Philadelphia chromosome-positive acute lymphoblastic leukemia: A GRAALL study. Biol Blood Marrow Transplant 19:150-155, 2013

4. Chalandon $Y$, Thomas $X$, Hayette $S$, et al: Randomized study of reduced-intensity chemotherapy combined with imatinib in adults with Ph-positive acute lymphoblastic leukemia. Blood 125:3711-3719, 2015
5. Brissot $E$, Labopin $M$, Beckers $M M$, et al: Tyrosine kinase inhibitors improve long-term outcome of allogeneic hematopoietic stem cell transplantation for adult patients with Philadelphia chromosome positive acute lymphoblastic leukemia. Haematologica 100:392-399, 2015

6. Soverini S, Colarossi S, Gnani A, et al: Resistance to dasatinib in Philadelphia-positive leukemia patients and the presence or the selection of mutations at residues 315 and 317 in the BCR-ABL kinase domain. Haematologica 92:401-404, 2007

7. Maino $E$, Sancetta R, Viero $P$, et al: Current and future management of Ph/BCR-ABL positive ALL. Expert Rev Anticancer Ther 14:723-740, 2014

8. Ottmann $O G$, Pfeifer $\mathrm{H}$ : Management of Philadelphia chromosome-positive acute lymphoblastic leukemia (Ph+ ALL). Hematology (Am Soc Hematol Educ Program) 2009:371-381, 2009

9. Leoni $\mathrm{V}$, Biondi $A$ : Tyrosine kinase inhibitors in BCR-ABL positive acute lymphoblastic leukemia. Haematologica 100:295-299, 2015

10. Lilly MB, Ottmann OG, Shah NP, et al: Dasatinib $140 \mathrm{mg}$ once daily versus $70 \mathrm{mg}$ twice daily in patients with Ph-positive acute lymphoblastic leukemia who failed imatinib: Results from a phase 3 study. Am J Hematol 85:164-170, 2010

11. Cortes JE, Kim DW, Pinilla-lbarz J, et al: A phase 2 trial of ponatinib in Philadelphia chromosome-positive leukemias. N Engl J Med 369:1783-1796, 2013

12. Radich JP: Molecular measurement of minimal residual disease in Philadelphia-positive acute lymphoblastic leukaemia. Best Pract Res Clin Haematol 15:91-103, 2002

13. Ravandi F, Jorgensen JL, Thomas DA, et al: Detection of MRD may predict the outcome of patients with Philadelphia chromosome-positive ALL treated with tyrosine kinase inhibitors plus chemotherapy. Blood 122:1214-1221, 2013

14. Scheuring UJ, Pfeifer $H$, Wassmann $B$, et al: Serial minimal residual disease (MRD) analysis as a predictor of response duration in Philadelphia-positive acute lymphoblastic leukemia $(\mathrm{Ph}+\mathrm{ALL})$ during imatinib treatment. Leukemia 17:1700-1706, 2003

15. Miller GD, Bruno BJ, Lim CS: Resistant mutations in $\mathrm{CML}$ and $\mathrm{Ph}(+) \mathrm{ALL}$ : Role of ponatinib. Biologics 8:243-254, 2014 
16. Zabriskie MS, Eide CA, Tantravahi SK, et al: BCR-ABL1 compound mutations combining key kinase domain positions confer clinical resistance to ponatinib in $\mathrm{Ph}$ chromosome-positive leukemia Cancer Cell 26:428-442, 2014

17. Deininger MW, Hodgson JG, Shah NP, et al: Compound mutations in BCR-ABL1 are not major drivers of primary or secondary resistance to ponatinib in CP-CML patients. Blood 127:703-712, 2016

18. Bargou R, Leo E, Zugmaier G, et al: Tumor regression in cancer patients by very low doses of a $T$ cell-engaging antibody. Science 321:974-977, 2008

19. Topp MS, Gökbuget N, Stein AS, et al: Safety and activity of blinatumomab for adult patients with relapsed or refractory B-precursor acute lymphoblastic leukaemia: A multicentre, single-arm, phase 2 study. Lancet Oncol 16:57-66, 2015

20. Topp MS, Gökbuget N, Zugmaier G, et al: Phase II trial of the anti-CD19 bispecific $T$ cellengager blinatumomab shows hematologic and molecular remissions in patients with relapsed or refractory B-precursor acute lymphoblastic leukemia. J Clin Oncol 32:4134-4140, 2014

21. van Rhee $F$, Hochhaus $A$, Lin $F$, et al: $p 190$ BCR-ABL mRNA is expressed at low levels in p210positive chronic myeloid and acute lymphoblastic leukemias. Blood 87:5213-5217, 1996

22. National Cancer Institute: $\mathrm{NCl}$ Common Terminology Criteria for Adverse Events (CTCAE) v4.0. Bethesda, MD, National Cancer Institute, 2014

23. Simon R: Optimal two-stage designs for phase II clinical trials. Control Clin Trials 10:1-10, 1989

24. Nicolini FE, Basak GW, Soverini $S$, et al: Allogeneic stem cell transplantation for patients harboring T315I BCR-ABL mutated leukemias. Blood 118:5697-5700, 2011
25. Soverini $S$, Gnani $A$, Colarossi $S$, et al: Philadelphia-positive patients who already harbor imatinib-resistant Bcr-Abl kinase domain mutations have a higher likelihood of developing additional mutations associated with resistance to second- or third-line tyrosine kinase inhibitors. Blood 114 2168-2171, 2009

26. Parker WT, Yeung DT, Yeoman AL, et al: The impact of multiple low-level BCR-ABL1 mutations on response to ponatinib. Blood 127:1870-1880, 2016

27. Ottmann OG, Larson RA, Kantarjian HM, et al: Phase II study of nilotinib in patients with relapsed or refractory Philadelphia chromosome-positive acute lymphoblastic leukemia. Leukemia 27:1411-1413, 2013

28. Ottmann $O$, Dombret $H$, Martinelli $G$, et al: Dasatinib induces rapid hematologic and cytogenetic responses in adult patients with Philadelphia chromosome positive acute lymphoblastic leukemia with resistance or intolerance to imatinib: Interim results of a phase 2 study. Blood 110:2309-2315, 2007

29. Cortes JE, Kantarjian $\mathrm{H}$, Shah NP, et al: Ponatinib in refractory Philadelphia chromosomepositive leukemias. N Engl J Med 367:2075-2088, 2012

30. Kantarjian H, Stein A, Gökbuget $N$, et al: Blinatumomab versus chemotherapy for advanced acute lymphoblastic leukemia. N Eng J Med 376:836847, 2017

31. Cortes-Franco J, Khoury HJ, Nicolini FE, et al: Safety and efficacy of subcutaneous-administered omacetaxine mepesuccinate in imatinib-resistant chronic myeloid leukemia (CML) patients who harbor the BCR-ABL T315I mutation: Results of an ongoing multicenter phase 2/3 study. Blood 114:644, 2009 (abstr)

32. Cortes J, Talpax M, Bixby D, et al: A phase I trial (AP 24534) in patients with refractory chronic myelogenous leukemia (CML) and other hematologic malignancies: Emerging safety and clinical response findings. Blood 116:210, 2010 (abstr)
33. Kantarjian HM, DeAngelo DJ, Stelljes M, et al: Inotuzumab ozogamicin versus standard therapy for acute lymphoblastic leukemia. N Engl J Med 375: 740-753, 2016

34. Kantarjian HM, Stein AS, Bargou RC, et al: Blinatumomab treatment of older adults with relapsed/ refractory B-precursor acute lymphoblastic leukemia: Results from two phase 2 studies. Cancer 122:21782185, 2016

35. Eide CA, O'Hare T: Chronic myeloid leukemia: Advances in understanding disease biology and mechanisms of resistance to tyrosine kinase inhibitors. Curr Hematol Malig Rep 10:158-166, 2015

36. Goldstone $A H$, Richards SM, Lazarus $H M$, et al: In adults with standard-risk acute lymphoblastic leukemia, the greatest benefit is achieved from a matched sibling allogeneic transplantation in first complete remission, and an autologous transplantation is less effective than conventional consolidation/maintenance chemotherapy in all patients: Final results of the International ALL Trial (MRC UKALL XII/ECOG E2993). Blood 111: 1827-1833, 2008

37. Bassan R, Spinelli O, Oldani E, et al: Improved risk classification for risk-specific therapy based on the molecular study of minimal residual disease (MRD) in adult acute lymphoblastic leukemia (ALL). Blood 113:4153-4162, 2009

38. Spinelli O, Peruta B, Tosi M, et al: Clearance of minimal residual disease after allogeneic stem cell transplantation and the prediction of the clinical outcome of adult patients with high-risk acute lymphoblastic leukemia. Haematologica 92:612-618, 2007

39. Bassan R, Spinelli O, Oldani E, et al: Different molecular levels of post-induction minimal residual disease may predict hematopoietic stem cell transplantation outcome in adult Philadelphia-negative acute lymphoblastic leukemia. Blood Cancer J 4: e225, 2014

\section{Affiliations}

Giovanni Martinelli and Cristina Papayannidis, Institute of Hematology and Medical Oncology "L. and A. Seràgnoli", Bologna; Alessandro Rambaldi, University of Milan Hematology and Bone Marrow Transplant Unit, Azienda Ospedaliera Papa Giovanni XXIII, Bergamo, Italy; Nicolas Boissel, University Paris Diderot, Hôpital Saint-Louis, Paris; Patrice Chevallier, Centre Hospitalier Universitaire Nantes, Nantes, France; Oliver Ottmann, Cardiff University, Cardiff; Adele K. Fielding, University College London Cancer Institute, London, United Kingdom; Nicola Gökbuget, University Hospital, Goethe University, Frankfurt; Max S. Topp, Universitätsklinikum Würzburg, Würzburg, Germany; Ellen K. Ritchie, Weill Cornell Medical College and New York Presbyterian Hospital, New York, NY; Lulu Ren Sterling, Amgen, San Francisco; Jonathan Benjamin, Amgen, Thousand Oaks; and Anthony Stein, Gehr Family Center for Leukemia Research, City of Hope, Duarte, CA.

Supported by Amgen.

\section{Support}

\section{Prior Presentation}

Presented in part as an oral presentation at the 57th American Society of Hematology Annual Meeting and Exposition, Orlando, FL, December 5-8, 2015. 


\section{AUTHORS' DISCLOSURES OF POTENTIAL CONFLICTS OF INTEREST}

Complete Hematologic and Molecular Response in Adult Patients With Relapsed/Refractory Philadelphia Chromosome-Positive B-Precursor Acute Lymphoblastic Leukemia Following Treatment With Blinatumomab: Results From a Phase II, Single-Arm, Multicenter Study

The following represents disclosure information provided by authors of this manuscript. All relationships are considered compensated. Relationships are self-held unless noted. I = Immediate Family Member, Inst = My Institution. Relationships may not relate to the subject matter of this manuscript. For more information about ASCO's conflict of interest policy, please refer to www.asco.org/rwc or ascopubs.org/jco/site/ifc.

Giovanni Martinelli

Consulting or Advisory Role: ARIAD Pharmaceuticals, Pfizer, Roche, Celgene

Speakers' Bureau: Novartis, Bristol-Myers Squibb

\section{Nicolas Boissel}

Honoraria: Amgen

Consulting or Advisory Role: Amgen

Patrice Chevallier

Consulting or Advisory Role: Amgen

\section{Oliver Ottmann}

Honoraria: Amgen, Novartis, Pfizer, Sunesis Pharmaceuticals, ARIAD Pharmaceuticals, Celgene, Fusion Pharma

Consulting or Advisory Role: Amgen, Novartis, Pfizer, ARIAD

Pharmaceuticals, Sunesis Pharmaceuticals, Celgene, Fusion Pharma

\section{Nicola Gökbuget}

Honoraria: Amgen, Pfizer

Consulting or Advisory Role: Amgen, Pfizer

Research Funding: Amgen, Pfizer

Travel, Accommodations, Expenses: Amgen, Pfizer

Max S. Topp

Honoraria: Amgen

Consulting or Advisory Role: Amgen

Speakers' Bureau: Amgen

Research Funding: Amgen

Travel, Accommodations, Expenses: Amgen

\section{Adele K. Fielding}

Consulting or Advisory Role: Amgen

Research Funding: Sigma Tau Pharmaceuticals

\section{Alessandro Rambaldi}

Honoraria: Roche, Amgen, Pierre Fabre

Consulting or Advisory Role: Genentech, Amgen

Ellen K. Ritchie

Consulting or Advisory Role: Incyte, Celgene, Pfizer, Novartis Speakers' Bureau: Celgene, Incyte, Novartis

Research Funding: Astellas Pharma (Inst), Bristol-Myers Squibb (Inst), Novartis (Inst), NS Pharma (Inst), Pfizer (Inst)

Travel, Accommodations, Expenses: Celgene, Novartis

Cristina Papayannidis

No relationship to disclose

Lulu Ren Sterling

Employment: Amgen

Stock or Other Ownership: Amgen

Jonathan Benjamin

Employment: Amgen

Stock or Other Ownership: Amgen

Travel, Accommodations, Expenses: Amgen

Anthony Stein

Consulting or Advisory Role: Amgen

Speakers' Bureau: Amgen

Research Funding: Amgen, Celgene, Argos, Seattle Genetics, Stemline Therapeutics 


\section{Acknowledgment}

We thank Heinz Horst, MD, PhD (Department of Internal Medicine II), and Wolfram Klapper, MD (Department of Pathology), University Hospital Schleswig-Holstein, Campus Kiel, Germany, for providing expert analysis of cytomorphology and histopathology, respectively, and Janice Y. Ahn, PhD, (Amgen) for medical writing assistance in the preparation of this manuscript.

\section{Appendix}

\section{METHODS}

Dose modifications, interruptions, and discontinuation. Blinatumomab was temporarily or permanently reduced to $9 \mu \mathrm{g} / \mathrm{day}$, if necessary, on the basis of the investigator's judgment for reasons of safety. Patients with dose reductions had the option to receive the higher dose level of $28 \mu \mathrm{g} /$ day once the adverse event (AE; except for neurologic events) resolved to at least grade 1 for $\geq 7$ days. Dexamethasone pretreatment was required for all patients who restarted blinatumomab treatment. For patients with signs of cytokine release syndrome, oral or intravenous dexamethasone $8 \mathrm{mg}$ was given three times per day for up to 3 days and reduced stepwise over 4 days. For grade $\geq 3$ cytokine release syndrome, tumor lysis syndrome, and disseminated intravascular coagulation/ coagulopathy, treatment was interrupted until the event resolved to at least grade 1 . For grade $\geq 3$ infections, blinatumomab was interrupted until the infection was adequately controlled or resolved per investigator opinion, and then allowed to restart at the lowest starting dose ( $9 \mu \mathrm{g} /$ day). Blinatumomab was permanently discontinued for grade 4 AEs that were possibly related to blinatumomab or for AEs that lasted 2 weeks or longer. For grade $\geq 3$ neurologic events, blinatumomab was stopped immediately, and the patient was assessed by physical examination, vital signs, and safety laboratories. To exclude potential infectious causes, cerebrospinal fluid was collected and assessed by cytology, cell count, B- and T-cell measurements, and viral studies. For grade 3 neurologic events or serious AEs leading to treatment interruption, treatment was restarted no earlier than 72 hours after stopping infusion but within 2 weeks. 